\title{
OCULAR ISCHEMIC SYNDROME: THE FIRST MANIFESTATION OF BILATERAL COMMON AND INTERNAL CAROTID ARTERIES OCCLUSION

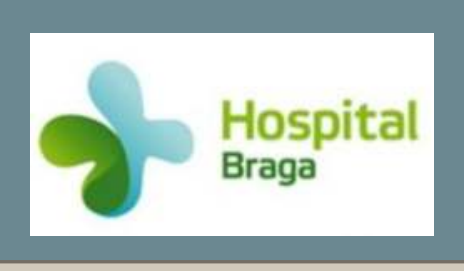

\author{
Inês Carvalho', Carla Ferreira ${ }^{1}$, João Pinho ${ }^{1}$, João Pereira ${ }^{1}$ \\ ${ }^{1}$ Neurology Department, Hospital de Braga, Portugal
}

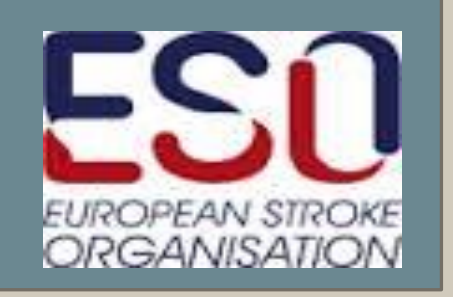

\section{BACKGROUND}

* Ocular Ischemic Syndrome (OIS) is a rare form of ischemia of both segments of the eye.

* It's caused by ocular hypoperfusion due to stenosis or occlusion of the carotid arteries mainly due to atherosclerosis.

* OIS as the first manifestation of bilateral carotid occlusion is rare.

\section{CASE REPORT}

* A 63-year-old man with history of smoking, excessive alcohol intake, dyslipidemia and polyglobulia, had progressive loss of vision for one month and bilateral periorbital pain in decubitus associated with conjunctival hyperemia for ten days.

* On the admission day, there was a short-lasting episode of altered state of consciousness associated with involuntary movements of the upper limbs, which had also occurred two days earlier.

* Neurological examination revealed a bilateral hypovision.

* Ophthalmologic examination showed a greatly reduced visual acuity in both eyes, medium mydriasis, rubeosis iridis, retinal microaneurysms and cotton-wool spots.

\section{INVESTIGATION}

CT showed two watershed infarcts in the right frontal and left parieto-occipital location.

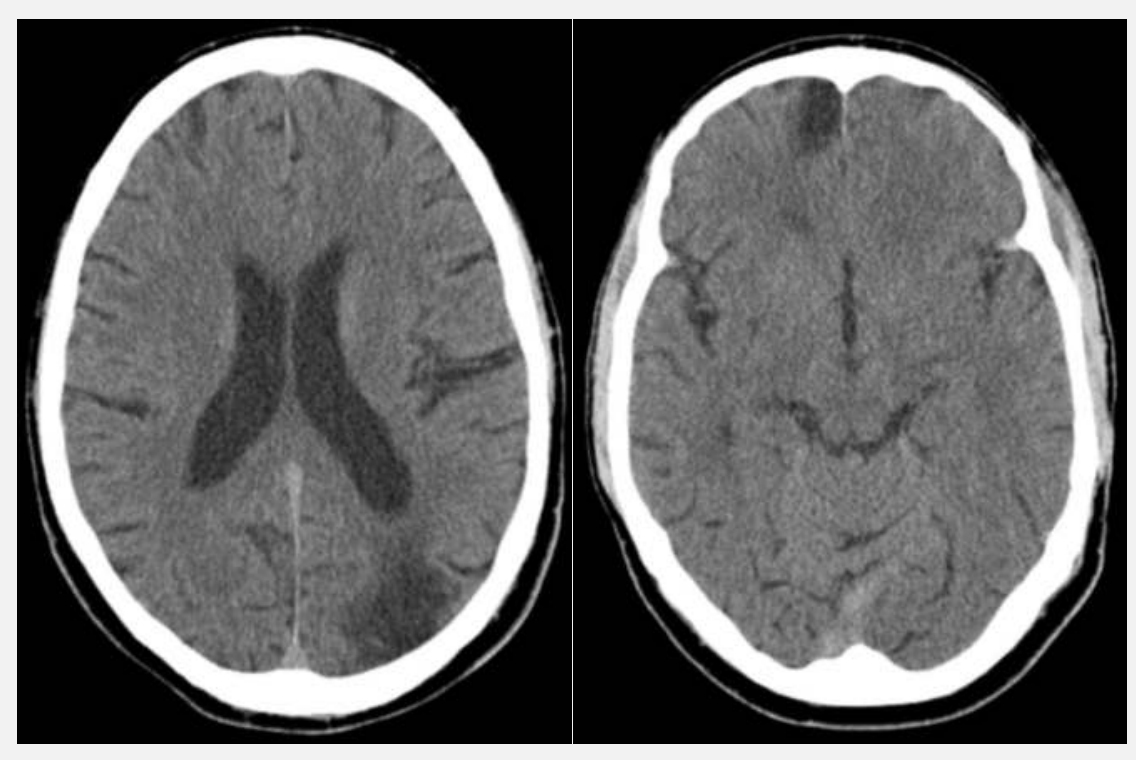

CT angiography revealed occlusion of both common and internal carotid arteries, without significant atherosclerotic aortic arch disease.
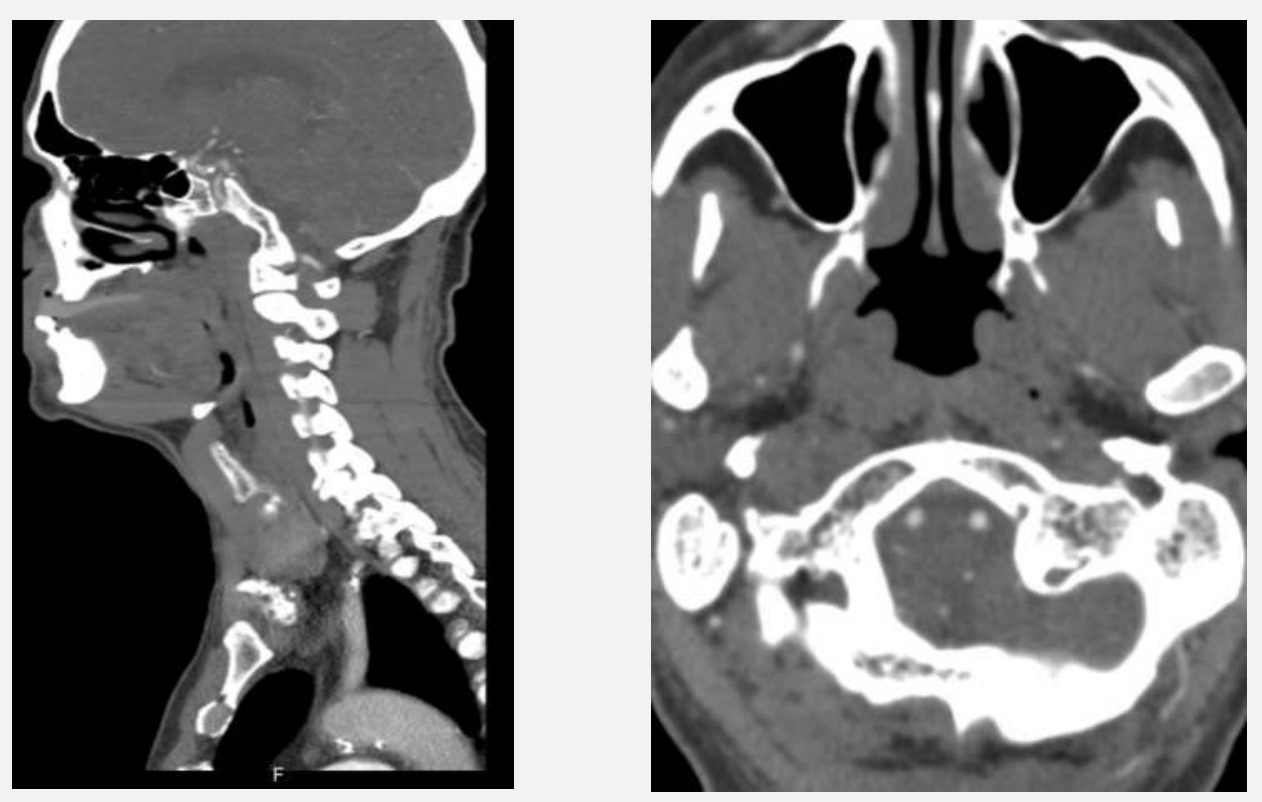

Doppler ultrassound confirmed severe atheromatous disease (a) and showed normal flow in external carotid arteries (ECA) (b) and inverted flow in ophthalmic arteries (c). ECA are supplied by anastomoses with the posterior circulation and thyroid branches.

a)

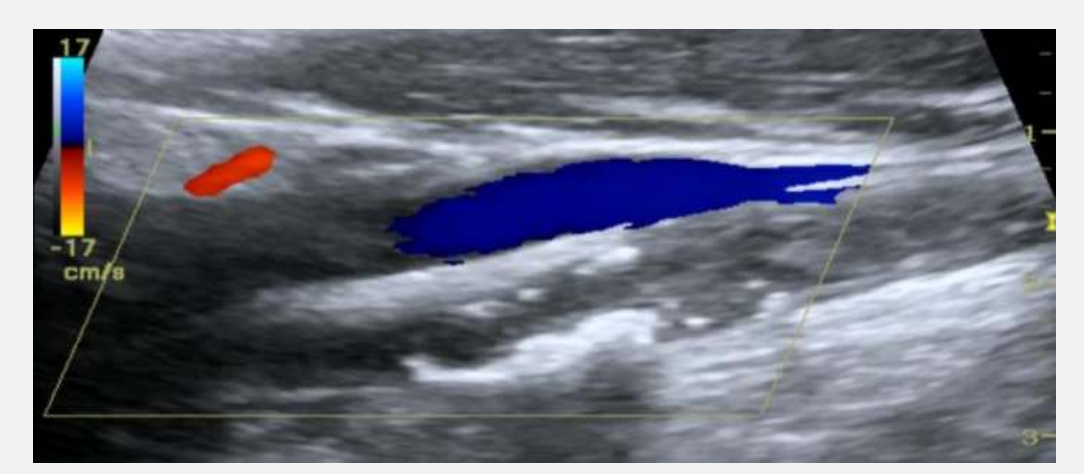

b)

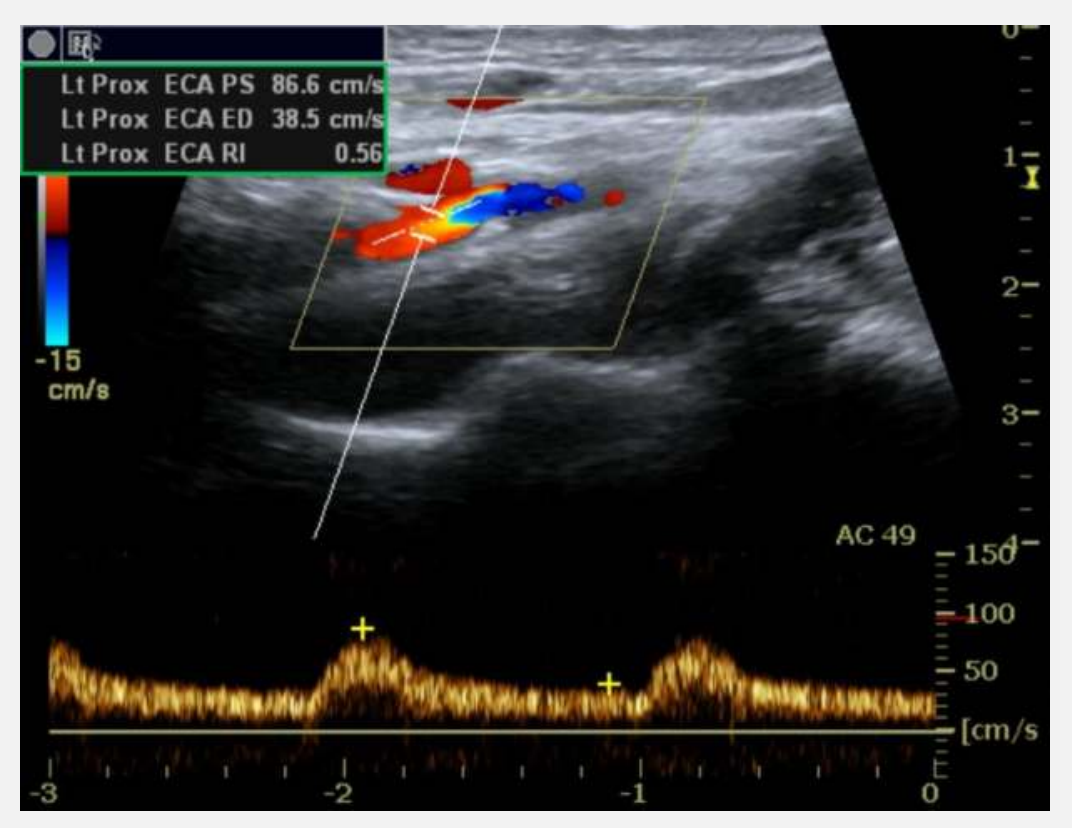

c)

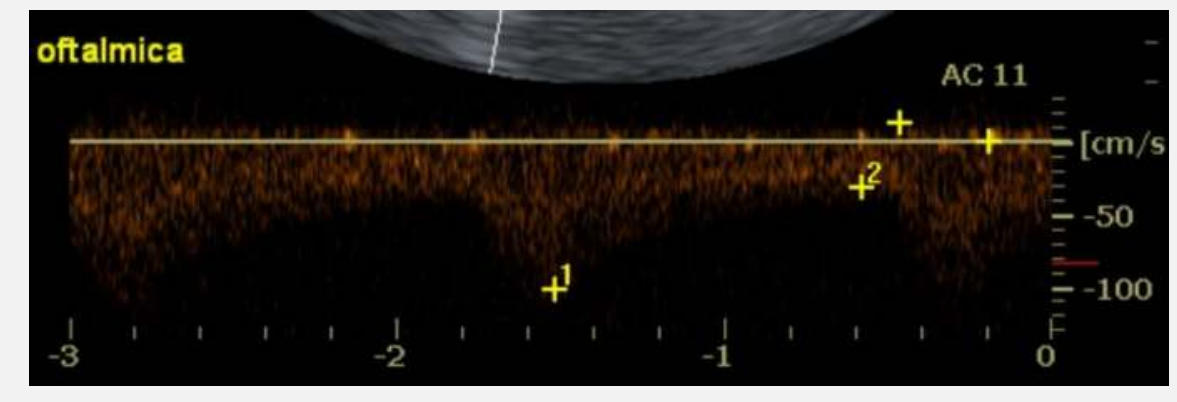

\section{CONCLUSIONS}

* Our patient presented an OIS as the first manifestation of bilateral occlusion of the carotid arteries. He was treated with dual antiplatelet therapy, high-dose statin and general recommendations for vascular risk factor control and hypotension prevention were made.

* It's important to alert that ocular manifestations may precede those of the cerebrovascular system, and thus, potentially life-threatening events can be prevented. 